\section{Gesellschaft für die Geschichte der Schweizer Psychiatrie und Psychotherapie (GGSP)}

Am 19. Januar 2001 wurde in Zürich die Gesellschaft für die Geschichte der Schweizer Psychiatrie und Psychotherapie (GGSP) in Zürich gegründet. Als Präsident wurde Prof. Dr. D. Hell gewählt und als Vorstandsmitglieder Dr. T. Hubschmid, Dr. B. Küchenhoff, PD Dr. Dr. A. Möller, Prof. Dr. Ch. Müller. Das Ziel der Gesellschaft ist die wissenschaftliche Erarbeitung der Schweizer Psychiatriegeschichte.

Jede an der Geschichte der Schweizer Psychiatrie interessierte Person kann Antrag auf Mitgliedschaft in die Gesellschaft stellen. Über die Annahme der Anträge entscheidet der Vorstand im Sinne der Vereinsstatuten, die auf Wunsch zugesandt werden. Der Mitgliedsbeitrag beträgt Fr. 50.- pro Jahr. Prof. Dr. med. D. Hell, Psychiatrische Universitätsklinik, Postfach 68, 8029 Zürich, Tel. 0138423 12, Fax 0138427 19, E-mail: hellsek@bli.unizh.ch

\section{Schweizerische Gesellschaft für Konsiliar- und Liaisonpsychiatrie (SGKLP)}

\section{Vorstand}

Präsident:

Dr. med. Franz Caduff, Thun

Kassier:

Dr. med. Urs Stillhard, St. Gallen

Sekretär:

Dr. med. Dan Georgescu, Brugg

Beisitzer.

Dr. med. Andreas Mohl, Baden;

Dr. med. Pascal Berger, Basel

Rechnungsrevisoren:

Dr. med. Sylvain Berner, Münchenstein;

Dr. med. Hans-Georg Kopp, Bellikon

\section{Schweizerische Gesellschaft für Kardiologie}

\section{Société Suisse de Cardiologie}

Vorstand/Comité

Präsident/Président:

Prof. Bernhard Meier, Bern

Past-President/Président sortant:

Prof. René Lerch, Genève

Vizepräsident/Vice-président:

Prof. Thomas F. Lüscher, Zürich
Sekretär/Secrétaire:

Prof. Peter Buser, Basel

Kassier/Trésorier:

Dr. Ezio Foglia, Massagno-Lugano

Mitglieder/Membres:

Dr. Klaus Ammann, Lichtensteig;

Prof. Osmund Bertel, Zürich;

Prof. Martin Fromer, Lausanne;

Dr Andres Jaussi, Yverdon-les-Bains;

Dr Michel Périat, Porrentruy;

Dr. Roberto Tartini; Zürich

Vertreter Kinderkardiologie /

Représentant Cardiologie pédiatrique:

Prof. Beat Friedli, Genève

Sekretariat/Secrétariat:

Dr Catherine Perrin, Montreux

(Geschäftsführerin)

Schweizerische Gesellschaft für Kardiologie / Société Suisse de Cardiologie,

Schwarztorstrasse 18, 3007 Bern,

Tel. 03138880 90, Fax 03138880 98,

E-mail: info@swisscardio.com,

Internet: http://www.swisscardio.com

\section{Schweizerische Gesellschaft für Infektiologie}

\section{Société Suisse d'Infectiologie}

Vorstand/Comité 2001

Präsident/Président:

Prof. Martin Täuber, Bern

Sekretär/Secrétaire:

Dr. Stephen Leib, Bern

Kassier/Trésorier:

Dr Hugo Sax, Genève

Mitglieder/Membres:

Prof. Bernard Hirschel, Genève;

Prof. Daniel Lew, Genève;

Prof. Philippe Moreillon, Lausanne;

Prof. David Nadal, Zürich;

Prof. Claude Regamey, Fribourg;

Prof. Urs Beat Schaad, Basel;

Dr. Claude Scheidegger, Basel;

Prof. Rainer Weber, Zürich;

PD Dr. Andreas Widmer, Basel

Sekretariat/Secrétariat:

Dr. Stephen Leib, Universität Bern, Institut für Infektionskrankheiten,

Friedbühlstrasse 51, Postfach 61,

3010 Bern, Tel. 03163249 49,

Fax 03163246 99, E-mail: info@sginf.ch, Internet: http://www.sginf.ch

\section{Schweizerische Gesellschaft für Nuklearmedizin}

Société Suisse de Médecine Nucléaire

Vorstand/Comité

Präsident/Président:

Prof. Dr. Dr. h.c. Johannes Locher, Aarau

Vize-Präsident/Vice-président:

Prof. Dr Angelika Bischof Delaloye, Lausanne

Sekretär/Secrétaire:

PD Dr. Hans Steinert, Zürich

Kassier/Trésorier:

Prof. Dr. Bernd Bubeck, St. Gallen

Beisitzer/Membres:

Dr. Andreas Haldemann, Bellinzona;

Dr. Friedrich Höflin, Chur;

Prof. Dr. Jan Müller-Brand, Basel

\section{Schweizerische Gesellschaft für Unterwasser- und Hyperbarmedizin SGUHM}

\section{Société suisse de médecine subaquatique et hyperbare SSMSH}

Vorstand/Comité

Präsident/Président:

Dr. med. Jörg Schmutz , Basel

Vize-Präsident/Vice-président: Dr. med. Claudio Camponovo, Lugano

Kassier/Trésorier:

Dr. med. Daniel Blickenstorfer, Zürich

Generalsekretär/Secrétaire général: Dr. med. Peter Knessl, Kilchberg

Wissenschaftliche Kommission / Commission scientifique: Prof. Dr Daniel Slosman, Genève

Organisationskommission / Commission d'organisation: Dr. med. Martin Kraus, Weinfelden

$P R$ :

Dr Guy de Watteville, Corgémont

Sekretariat/Secrétariat:

c/o M. Spahr,

Lerchenweg 9, 2543 Lengnau, Tel. 03265385 46, Fax 0326538547 E-mail:suhms@datacomm.ch, Internet: http://www.suhms.org 


\section{Wichtige Mitteilung}

Erhöhtes Rhabdomyolyserisiko bei der Kombination von Cerivastatin mit Gemfibrozil

\section{Neue Kontraindikation}

für LIPOBAY ${ }^{\circledR} 0,1 / 0,2 / 0,3 / 0,4 \mathrm{mg}$,

Vertrieb Bayer (Schweiz) AG, und LIPIQOL ${ }^{\circledR} 0,1 / 0,2 / 0,3 / 0,4 \mathrm{mg}$,

Vertrieb Takeda Pharma AG

(Wirkstoff: Cerivastatin)

\section{Sehr geehrte Damen und Herren}

Wir informieren Sie hiermit über eine wichtige vorgesehene Änderung in der Fachinformation $\mathrm{zu}$ LIPOBAY ${ }^{\circledR}$ und LIPIQOL ${ }^{\circledR}$ $0,1 / 0,2 / 0,3 / 0,4 \mathrm{mg}$, die die Anwendung dieser Arzneimittel betrifft. LIPOBAY ${ }^{\circledR}$ und LIPIQOL ${ }^{\circledR}$ sind indiziert zur Behandlung von Patienten mit primärer Hypercholesterinämie Typ IIA + Typ IIB (ohne/mit Hypertriglyzeridämie), wenn Diät allein eine ungenügende Wirkung gezeigt hat.

Die neue Fachinformation zu LIPOBAY ${ }^{\circledR /}$ LIPIQOL ${ }^{\circledR}$ wird die gleichzeitige Anwendung von Cerivastatin mit Gemfibrozil als Kontraindikation ausweisen.

Bisher wurde im Kapitel "Vorsichtsmassnahmen" empfohlen, diese Kombination nicht einzusetzen.

Patienten, die gleichzeitig LIPOBAY® bzw. LIPIQOL ${ }^{\circledR}$ und Gemfibrozil erhalten, müssen unverzüglich eine dieser Therapien beenden. Die anschliessende Behandlung dieser Patienten sollte auf ihre individuellen Bedürfnisse zugeschnitten sein.

Eine Myopathie oder eine Rhabdomyolyse können in Verbindung mit der Anwendung von HMG-CoA-Reduktasehemmern, einschliesslich Cerivastatin, und Fibraten auftreten. Spontanberichte unerwünschter Arzneimittelwirkungen haben ein erhöhtes Risiko für die Auslösung von Rhabdomyolysen bei gleichzeitiger Anwendung von LIPOBAY ${ }^{\circledR} / L^{2} I P I Q O L^{\circledR} \quad$ (Cerivastatin) und Gemfibrozil gezeigt. Pharmakokinetische Untersuchungen haben gezeigt, dass bei gleichzeitiger Gabe von Gemfibrozil die AUC von Cerivastatin aufs 4 fache ansteigt. Eine Rhabdomyolyse ist ein potentiell lebensbedrohlicher Zustand, der durch deutlich erhöhte CPK-Spiegel, Myoglobin im Serum und Myoglobinurie gekennzeichnet ist und zu akutem Nierenversagen führen kann.

Patienten, die LIPOBAY ${ }^{\circledR} /$ LIPIOOL $^{\circledR}$ (Cerivastatin) einnehmen, sollten auf diese mögliche potentiell lebensbedrohliche Nebenwirkung aufmerksam gemacht und angewiesen werden, ihren Arzt unverzüglich über unerklärliche Muskelschmerzen und Muskelschwäche zu informieren.
Die aktuelle Patienteninformation weist die Patienten an, ihren Arzt zu kontaktieren, sobald sie eines der genannten Symptome wahrnehmen. LIPOBAY ${ }^{\circledR} /$ LIPIQOL $^{\circledR}$ muss unverzüglich abgesetzt werden, wenn deutlich erhöhte CPK-Spiegel gemessen oder eine Myopathie diagnostiziert oder vermutet werden.

Das Risiko, unter Therapie mit HMG-CoAReduktasehemmern eine Rhabdomyolyse zu entwickeln, ist vermutlich von der Plasmakonzentration dieser Wirkstoffe abhängig. Die benötigte Dosierung von LIPOBAY ${ }^{\circledR} /$ LIPIQOL ${ }^{\circledR}$ muss individuell gemäss dem therapeutischen Ansprechen festgelegt werden. Die Anfangsdosis beträgt 0,1 mg $1 \times$ täglich. In Abständen von mindestens 4 Wochen kann die Dosis schrittweise um 0,1 mg erhöht werden. Die höchste empfohlene Dosis beträgt 0,4 mg $1 \times$ täglich.

Obgleich die gleichzeitige Anwendung von Gemfibrozil und Cerivastatin in der Schweiz selten erfolgt, hat Bayer Pharma eigenverantwortlich bei der IKS eine Änderung der Fachinformation eingeleitet mit dem Ziel, den in der bisherigen Fachinformation enthaltenen Text zur gleichzeitigen Anwendung von Cerivastatin mit Gemfibrozil im Abschnitt "Vorsichtsmassnahmen" zu verstärken und als absolute Kontraindikation auszuweisen. Die Patientensicherheit ist Bayer Pharma stets ein vorrangiges Anliegen; daher informieren wir die medizinischen Fachkreise proaktiv über diese in die Wege geleitete wichtige Änderung.

Bayer Pharma beobachtet fortlaufend die Sicherheit ihrer Arzneimittel und ist weiterhin davon überzeugt, dass LIPOBAY ${ }^{\circledR /}$ LIPIQOL ${ }^{\circledR}$ (Cerivastatin) bei bestimmungsgemässer Anwendung wirksam und sicher ist.

Meldungen über unerwünschte Wirkungen bitten wir Sie zu richten an Bayer (Schweiz) AG, Takeda Pharma AG oder auf dem neuen gelben Meldeformular an das regionale Pharmacovigilance-Zentrum. Das Meldeformular ist verfügbar auf der Homepage der IKS (www.iks.ch unter "Heilmittelregistrierung, Formulare, Meldung einer vermuteten unerwünschten Arzneimittelwirkung") oder kann bei der IKS bestellt werden (Tel. 031 32202 23,E-mail:info@iks.admin.ch).

Wir bitten Sie um Ihre Kenntnisnahme. Mit freundlichen Grüssen

Bayer (Schweiz) AG

Dr. H. Braun

Leiter klinische Forschung

Takeda Pharma AG

Dr. R. Roten

Med. Leiterin, Division Kardiologie

\section{Avis important}

Risque accru de rhabdomyolyse de cérivastatine en association avec Gemfibrozil

Nouvelle contre-indication LIPOBAY ${ }^{\circledR} 0,1 / 0,2 / 0,3 / 0,4 \mathrm{mg}$, Distribution Bayer (Schweiz) $A G$, et IIPIQOL $^{\circledR} 0,1 / 0,2 / 0,3 / 0,4 \mathrm{mg}$, Distribution Takeda Pharma AG (principe actif: cérivastatine)

\section{Mesdames, Messieurs,}

Nous vous communiquons par la présente une modification importante prévue dans l'information scientifique sur LIPOBAY ${ }^{\circledR}$ et LIPIQOL ${ }^{\circledR} 0,1 / 0,2 / 0,3 / 0,4 \mathrm{mg}$ concernant l'indication à ces médicaments. LIPOBAY ${ }^{\circledR}$ et LIPIQOL ${ }^{\circledR}$ sont indiqués pour le traitement de l'hypercholestérolémie primaire de type IIA + type IIB (avec/sans hypertriglycéridémie), lorsque le régime seul n'a pas démontré un effet suffisant.

La nouvelle information scientifique sur LIPOBAY ${ }^{\circledR} /$ LIPIQOL $^{\circledR}$, précisera que l'administration en parallèle de cérivastatine et de gemfibrozil est une contre-indication.

Jusqu'ici, dans l'information scientifique sur LIPOBAY ${ }^{\circledR} /$ LIPIQOL $^{\circledR}$, au chapitre "Précautions", il était recommandé de ne pas recourir à cette association.

Les patients recevant IIPOBAY $^{\circledR} / \mathrm{LIPIQOL}^{\circledR}$ et gemfibrozil doivent absolument interrompre l'un de ces médicaments. La suite du traitement de ces patients doit être adaptée $\dot{a}$ leurs besoins individuels.

Une myopathie ou une rhabdomyolyse peut apparaître avec l'application d'inhibiteurs de l'HMG-CoA-réductase, cérivastatine y compris, et des fibrates. Des déclarations spontanées d'effets médicamenteux indésirables ont fait état d'un risque accru de rhabdomyolyse lors de l'administration concomitante de LIPOBAY®/LIPIQOL ${ }^{\circledR}$ (cérivastatine) et de gemfibrozil. Des examens pharmacocinétiques ont montré qu'avec la prise concomitante de gemfibrozil, l'AUC de la cérivastatine et 4 fois plus élevé. Une rhabdomyolyse est une situation potentiellement dramatique, caractérisée par une ascension des concentrations de CPK, la présence de myoglobine dans le sérum et une myoglobinurie, pouvant être à l'origine d'une insuffisance rénale aiguë. Les patients prenant LIPOBAY ${ }^{\circledR} /$ LIPIQOL $^{\circledR}$ (cérivastatine) doivent être rendus attentifs à cet effet indésirable potentiellement périlleux, et à consulter sans tarder leur médecin dès qu'ils ressentent des douleurs et une faiblesse musculaires inexplicables. 
L'information destinée aux patients leur dit de consulter leur médecin dès qu'ils ressentent l'un des symptômes cités. LIPOBAY ${ }^{\circledR /}$ LIPIQOL ${ }^{\circledR}$ doit être arrêté immédiatement dès que le dosage des CPK donne un résultat nettement pathologique, ou dès qu'une myopathie est diagnostiquée ou même suspectée. Le risque de présenter une rhabdomyolyse sous traitement par inhibiteurs de l'HMGCoA-réductase est supposé dépendre de la concentration plasmatique de ces substances. La posologie nécessaire de LIPOBAY $^{\circledR} /$ LIPIQOL ${ }^{\circledR}$ doit être adaptée individuellement en fonction du traitement et de la réponse du patient. La dose initiale et de 0,1 mg une fois par jour. Cette dose peut être augmentée par étapes de 0,1 mg par intervalles d'au moins 4 semaines. La dose maximale recommandée est de 0,4 mg une fois par jour. Malgré le fait que l'administration en parallèle de gemfibrozil et de cérivastatine soit plutôt rare en Suisse, Bayer Pharma a spontanément proposé une modification de l'information scientifique à l'OICM, dans le but de faire mieux ressortir le texte actuel sur l'administration concomitante de cérivastatine et de gemfibrozil au paragraphe "Précautions", et de la faire figurer dans les contre-indications absolues. La sécurité des patients a toujours été pour Bayer Pharma un devoir prioritaire; c'est la raison pour laquelle nous informons maintenant déjà le corps médical de la mise en route de cette adaptation importante.

Bayer Pharma suit constamment la sécurité de ses médicaments et reste convaincue qu'utilisé conformément aux prescriptions, LIPOBAY ${ }^{\circledR} /$ LIPIQOL $^{\circledR}$ (cérivastatine) est un médicament efficace et sûr.

Nous prions les professionnels de la santé d'adresser les rapports d'effets indésirables suspectés à Bayer (Schweiz) AG, Takeda Pharma AG ou au centre régional de pharmacovigilance indiqué sur le nouveau formulaire de déclaration. Ce formulaire est disponible sur le site internet de l'OICM (www.oicm.ch $\rightarrow$ enregistrement des médicaments, formulaires, annonce d'effets indésirables suspectés). Il peut aussi être commandé par téléphone (031 32202 23) ou pare-mail (info@iks.admin.ch).

Nous vous remercions de prendre bonne note de ce qui précède.

Avec nos meilleures salutations

Bayer (Schweiz) AG

Dr H. Braun

Chef de la recherche clinique

Takeda Pharma AG

Dr R. Roten

Responsable du département médical, division cardiologie

\section{Chargenrückruf}

Floxapen 500 mg Kapseln,

Packungsgrösse à 16 Kapseln,

IKS-Nr. 37'405, Charge 72763A

Bei Floxapen 500 mg Kapseln, Packungsgrösse à 16 Kapseln, der Charge 72763A (Verfall 09/2003) wurde vereinzelt in der Faltschachtel eine falsche Packungsbeilage vorgefunden.

Anstelle der Packungsbeilage für "Floxapen» liegt eine griechische Packungsbeilage für Augmentin $625 \mathrm{mg}$ vor.

Bei den Kapseln in den Blister handelt es sich um Floxapen 500 mg Kapseln, sodass bei der Anwendung der Kapseln kein besonderes Sicherheitsrisiko besteht.

Im Zeitraum zwischen dem 12. Juni 2001 und dem 17. Juli 2001 wurden 2670 Packungen dieser Charge 72763A über die Alloga AG, Burgdorf, an 23 Spitäler und 15 Grossisten, ausgeliefert.

Wir bitten Sie, sämtliche Lagerbestände von Floxapen $500 \mathrm{mg}$, Packungsgrösse à 16 Kapseln, der Charge 72763A, bezüglich Packungsbeilage zu kontrollieren und fehlerhafte Packungen vor dem 31. August 2001 an die Alloga AG, Buchmattstrasse 10, 3400 Burgdorf, oder Ihren Grossisten (gemäss Lieferweg) zu retournieren.

Die retournierten Packungen werden vollumfänglich ersetzt.

Wir entschuldigen uns für die entstehenden Umtriebe und danken für Ihr Verständnis. SmithKline Beecham AG, Brunnmattstrasse 13, 3174 Thörishaus, Tel. 03188821 48, Fax 0318882144

\section{Rappel de lot}

Floxapen capsules à $500 \mathrm{mg}$, emballages de 16 capsules, OICM $n^{0} 37^{\prime} 405, \operatorname{lot} \mathbf{n}^{0} 72763 \mathrm{~A}$

Dans des cas isolés, de fausses notices d'emballage ont été trouvées dans les conditionnements de Floxapen $500 \mathrm{mg}$, emballages de 16 capsules, lot $n^{0} 72763 A$ (date de péremption 09/2003).

A la place de la notice d'emballage «Floxapen" se trouve une notice d'emballage en grec pour l'Augmentin $625 \mathrm{mg}$.

Les capsules contenues dans les blisters sont effectivement des capsules de Floxapen 500 mg et leur usage ne représente donc aucun risque particulier en terme de sécurité.

Entre le 12 juin 2001 et le 17 juillet 2001, 2670 conditionnements du lot $n^{0} 72763 \mathrm{~A}$ ont été distribués par Alloga SA, Burgdorf, à 23 hôpitaux et 15 grossistes.

Nous vous prions de vérifier les conditionnements des capsules de Floxapen $500 \mathrm{mg}$, emballages de 16 capsules portant le lot $n^{0}$ 72763A que vous pourriez avoir en stock et de contrôler les notices d'emballage. Veuillez retourner les conditionnements non conformes avant le 31 août 2001 à Alloga SA, Buchmattstrasse 10, 3400 Burgdorf, ou à votre grossiste (selon votre mode de livraison).

Les conditionnements retournés seront entièrement remplacés.

Nous vous prions de nous excuser pour ces désagréments et vous remercions de votre compréhension.

SmithKline Beecham AG, Brunnmattstrasse 13, 3174 Thörishaus, Tel. 03188821 48, Fax 0318882144. 


\section{Medikamentenrückruf}

Diprophos Einwegspritze $1 \mathrm{ml}$, Batch 0006 IKS Nr. 39'528

Sehr geehrte Damen und Herren

Möglicherweise enthalten einige der Diprophos Einwegspritzen $(1 \mathrm{ml})$ des Batches 0006 schwarze Partikel, weshalb wir den ganzen Produktionsbatch zurückrufen. Betroffen sind maximal 906 dieser Einwegspritzen, die vom 7. März 2001 bis zum 31. Mai, 2001 an Grossisten, Spitäler und Apotheken ausgeliefert wurden.

Aus Sicherheitsgründen bitten wir Sie daher unverzüglich um folgende Massnahmen:

1. Überprüfung Ihrer Lagerbestände auf Diprophos Einwegspritzen (1 ml), Charge 0006. Falls Lagerbestände noch vorhanden sind, bitten wir um unverzügliche Sperrung, damit keine weiteren Verteilungen stattfinden können.

2. Wir bitten Sie, allfällige Packungen direkt an Essex Chemie AG, Tribschenstrasse 11, 6002 Luzern, zurückzusenden. Wir werden Ihnen diese unentgeltlich ersetzen.

3. Sollten im Rahmen Ihrer Chargenüberprüfung Fragen auftreten, so wenden Sie sich direkt an den rechts Unterzeichnenden unter Tel. 04136849 20. Wir stehen Ihnen selbstverständlich jederzeit zur Verfügung.

Wir danken Ihnen im voraus für Ihre kooperative Mitarbeit und Unterstützung in dieser Angelegenheit.

Mit freundlichen Grüsse

ESSEX Chemie AG

\section{S. Sandor Dr. H. Egger}

General Manager Medizinischer Direktor

\section{Rappel de médicament}

Diprophos seringue à usage unique $1 \mathrm{ml}$, Batch 0006 Numéro IKS 39'528

Mesdames, Messieurs

Il est possible que quelques seringues à usage unique $(1 \mathrm{ml})$ de notre médicament Diprophos, du batch 0006 contiennent des particules noires, pour cette raison nous faisons un rappel, du batch complet de ce produit. Au maximum 906 pièces de ces seringues à usage unique, qui ont été livré aux grossistes, hôpitaux et pharmacies pendant la période du 7 mars 2001 jusqu'au 31 mai 2001, sont affectées.

Pour des raisons de sécurité, nous vous prions de prendre immédiatement les mesures suivantes:

1. Vérification de votre stock de Diprophos seringue à usage unique (1 $\mathrm{ml}$ ), charge 0006. Si vous avez encore du Diphrophos susmentionné en stock, nous vous prions de faire un blocage immédiate, afin d'éviter que le médicament soit distribué.

2. Au cas où, vous avez des emballages susmentionnés dans votre stock de marchandises, nous vous prions de les renvoyer directement à Essex Chimie SA, Tribschenstrasse 11, 6002 Lucerne. Ces emballages vous seront remplacés gratuitement.

3. Si vous avez des questions en ce qui concerne ce contrôle des charges, nous vous prions d'entrer en contact directement avec notre Directeur médical, le Docteur Hanspeter Egger, sous le numéro de téléphone suivant: 04136849 20, qui reste volontiers à votre disposition.

Nous vous remercions en avance, pour votre collaboration et soutien coopératif dans cette affaire, et vous envoyons, Mesdames, Messieurs, nos salutations les meilleures.

Essex Chimie SA

$\begin{array}{ll}\text { S. Sandor } & \text { Dr. H. Egger } \\ \text { General Manager } & \text { Directeur médical }\end{array}$

